EPJ Web of Conferences 13, 04001 (2011)

DOI: $10.1051 /$ epjconf/20111304001

C Owned by the authors, published by EDP Sciences, 2011

\title{
Charmonium production and elliptic flow in relativistic heavy-ion collisions
}

\author{
Che Ming $\mathrm{Ko}^{1, \mathrm{a}}$, Su Houng Lee ${ }^{2, \mathrm{~b}}$, Taesoo Song ${ }^{3, \mathrm{c}}$, and Jun $\mathrm{Xu}^{3, \mathrm{~d}}$ \\ 1 Cyclotron Institute and Department of Physics and Astronomy, Texas A\&M University, College Station, TX 77843-3366, \\ USA \\ 2 Institute of Physics and Applied Physics, Yonsei University, Seoul 120-749, Korea \\ 3 Cyclotron Institute, Texas A\&M University, College Station, TX 77843-3366, USA
}

\begin{abstract}
Using the two-component model for charmonium production, which includes contributions from both initial hard nucleon-nucleon scattering and from regeneration in the quark-gluon plasma, we have studied the nuclear modification factor $R_{A A}$ and elliptic flow $v_{2}$ of $J / \psi$ in relativistic heavy-ion collisions. For the expansion dynamics of produced hot dense matter, we have introduced a schematic fire-cylinder model with its transverse acceleration determined from the pressure gradient inside the fire-cylinder and azimuthally anisotropic expansion parameterized to reproduce measured $v_{2}$ of light hadrons. We have assumed that light hadrons freeze out at the temperature of $120 \mathrm{MeV}$ while charmonia at $160 \mathrm{MeV}$, similar to the kinetic and chemical freeze-out temperatures in the statistical model, respectively. For the properties of charmonia in the quark-gluon plasma, we have used the screening mass between their charm and anticharm quarks and their dissociation cross sections given by the perturbative QCD (pQCD) in the leading order and up to the next-to-leading order, respectively. For the relaxation time of charm and anticharm quarks in the quark-gluon plasma, we have also used the one calculated in the leading order pQCD. Modeling the effect of higher-order corrections in pQCD by introducing multiplicative factors to the dissociation cross sections of charmonia and the elastic scattering cross sections of charm and anticharm quarks, we have found that this effect is small for the $R_{A A}$ of $J / \psi$ as they suppress the number of initially produced $J / \psi$ but enhance the number of regenerated ones. The higher-order corrections increase, however, the $v_{2}$ of $J / \psi$. Our results thus suggest that the $v_{2}$ of $J / \psi$ can play an important role in discriminating between $J / \psi$ production from initial hard collisions and from regeneration in the quark-gluon plasma.
\end{abstract}

\section{Introduction}

One of the signatures suggested for the formation of the quark-gluon plasma (QGP) in relativistic heavy-ion collisions is the suppressed production of $J / \psi$ compared with that expected from initial hard scattering [1]. Experimentally, suppressed production of $J / \psi$ in heavy-ion collisions has been observed at both Super Proton Synchrotron (SPS) [2,3] and Relativistic Heavy Ion Collider (RHIC) [4,5]. Many theoretical explanations have been proposed to understand these experimental results [6-9]. Among them are the statistical model [9] and the two-component model [7]. In the statistical model, it is assumed that all initially produced $J / \psi$ are dissociated in QGP and the detected ones are entirely due to regeneration from charm and anticharm quarks in the QGP [9]. In the two-component model, the number of regenerated $J / \psi$ is smaller than that in the statistical model as some initially produced $J / \psi$ can survive during the collision if the $J / \psi$ dissociation temperature in QGP is sufficiently high [7]. Both models are able to successfully reproduce the measured nuclear modification fac-

\footnotetext{
a e-mail: ko@comp.tamu.edu

b e-mail: suhoung@yonsei.ac.kr

c e-mail: songtsoo@yonsei.ac.kr

d e-mail: xujun@comp.tamu.edu
}

tor of $J / \psi$ as a function of the number of participants [7, $9,10]$ and their transverse momentum $[11,12]$. Although the transverse momentum of thermally produced $J / \psi$ in the statistical model is smaller than that of $J / \psi$ produced from initial hard scattering, it is enhanced by the transverse flow. Therefore, it is difficult to discriminate the two models based on the number of finally produced $J / \psi$ and their transverse momentum distribution. Since initially produced $J / \psi$ have azimuthally isotropic transverse momenta, they are expected to have vanishing elliptic flow, which is in contrast with the appreciable elliptic flow of regenerated ones, the elliptic flow of $J / \psi$ in heavy ion collisions is thus sensitive to the fraction of $J / \psi$ that is produced from the regeneration in the QGP. Studying the elliptic flow of $J / \psi$ thus can help to discriminate between the two $J / \psi$ production mechanisms of from initial hard scattering and from regeneration in the QGP.

In this talk, we report results from our recent work on $J / \psi$ production and elliptic flow in heavy ion collisions at RHIC using the two-component model [13]. The talk is organized as follows. We briefly describe in Sec. 2 the two component model and in Sec. 3 the schematic model for the expanding fire-cylinder produced in heavy-ion collisions. In Sec. 4 , the nuclear modification factor of $J / \psi$ as a function of the number of participants and the transverse

This is an Open Access article distributed under the terms of the Creative Commons Attribution-Noncommercial License 3.0, which permits unrestricted use, distribution, and reproduction in any noncommercial medium, provided the original work is properly cited. 
momentum of $J / \psi$ are studied in this schematic model. Results on the elliptic flow of $J / \psi$ are presented in Sec. 5. Finally, the summary and conclusions are given in Sec. 6.

\section{2 the two-component model for $J / \psi$ production}

In the two-component model for $J / \psi$ production in relativistic heavy-ion collisions, some initially produced $J / \psi$ from hard scattering can survive through the collisions and contribute to the final observed $J / \psi$ in experiments, besides those regenerated from charm and anticharm quarks in the produced QGP.

\section{1 directly produced $J / \psi$}

The number of initially produced $J / \psi$ in a heavy-ion collision at impact parameter $b$ can be determined from the Glauber model as

$$
N_{J / \psi}^{A A}=\sigma_{J / \psi}^{N N} A^{2} T_{A A}(\mathbf{b}),
$$

in terms of the cross section $\sigma_{J / \psi}^{N N}$ for $J / \psi$ production in a $p+p$ collision, which has a value of $0.774 \mu \mathrm{b}$ per unit rapidity near midrapidity at collision energy $\sqrt{s}=200$ $\mathrm{GeV}$ [14], the mass number $A$ of colliding nuclei, and the nuclear overlap function

$$
T_{A A}=\int d^{2} s T_{A}(\mathbf{s}) T_{A}(\mathbf{b}-\mathbf{s}),
$$

where

$$
T_{A}(\mathbf{s})=\int_{-\infty}^{\infty} d z \rho_{A}(\mathbf{s}, z)
$$

is the thickness function with $\rho_{A}(\mathbf{s}, z)$ being the nucleon distribution in the colliding nucleus.

The survival probability of an initially produced $J / \psi$ in relativistic heavy-ion collisions is given by

$$
S_{\mathrm{th}}(\mathbf{b}, \mathbf{s})=\exp \left\{-\int_{\tau_{0}}^{\tau_{\mathrm{cf}}} \Gamma\left(\tau^{\prime}\right) d \tau^{\prime}\right\}
$$

where $\tau_{0}$ is the time the $J / \psi$ is produced from an initial hard scattering and $\tau_{\mathrm{cf}}$ is the time the $J / \psi$ freezes out from the hot dense matter. The $J / \psi$ survival probability depends on its thermal decay width $\Gamma(T)$ in the produced hot dense matter,

$$
\Gamma(T)=\sum_{i} \int \frac{d^{3} k}{(2 \pi)^{3}} v_{\mathrm{rel}}(k) n_{i}(k, T) \sigma_{i}^{\mathrm{diss}}(k, T) .
$$

In the above, the summation is over the particle species $i$, which includes the quarks and gluons in QGP as well as the baryons and mesons in the hadron gas, with its density denoted by $n_{i}(k, T)$; $v_{\text {rel }}$ is the relative velocity between the $J / \psi$ and the particle. For the dissociation cross section $\sigma_{i}^{\text {diss }}$ of $J / \psi$, it is calculated in pQCD up to the next-to-leading order if it is in QGP and is calculated via the factorization formula with the Bethe-Salpeter amplitude for the bound charmonium states if it is in the hadron gas $[15,16]$.

Since some directly produced $J / \psi$ are from the decay of directly produced excited charmonium states $\chi_{c}$ and $\psi^{\prime}$, the survival probability of an initially produced $J / \psi$ from thermal decay is the linear combination of the survival probabilities for $J / \psi, \chi_{c}$, and $\psi^{\prime}$, that is

$$
S_{\mathrm{th}}(\mathbf{b}, \mathbf{s})=0.67 S_{\mathrm{th}}^{J / \psi}(\mathbf{b}, \mathbf{s})+0.25 S_{\mathrm{th}}^{\chi_{c}}(\mathbf{b}, \mathbf{s})+0.08 S_{\mathrm{th}}^{\psi^{\prime}}(\mathbf{b}, \mathbf{s}),
$$

where contributions to $J / \psi$ production from the feed-down of $\chi_{c}$ and $\psi^{\prime}$ are taken to be $25 \%$ and $8 \%$, respectively, as usually adopted in the literature [17].

The directly produced $J / \psi$, which is produced by gluon fusions, is affected by the Cronin effect due to the gluonnucleon scattering. This leads to an increase of the average squared transverse momentum $\left\langle p_{T}^{2}\right\rangle_{A A}$ of $J / \psi$ in A+A collisions, i.e.,

$$
\left\langle p_{T}^{2}\right\rangle_{A A}=\left\langle p_{T}^{2}\right\rangle_{p p}+\frac{\left\langle\delta p_{T}^{2}\right\rangle}{\lambda_{g N}}\left\langle L_{g N}\right\rangle,
$$

where $\left\langle p_{T}^{2}\right\rangle_{p p}$ is the average squared transverse momentum of $J / \psi$ in $p+p$ collisions, $\left\langle\delta p_{T}^{2}\right\rangle$ is the average of added squared transverse momentum by one gluon-nucleon scattering, and $\lambda_{g N}$ and $\left\langle L_{g N}\right\rangle$ are, respectively, the mean free path and average path length of the gluon in uniform nuclear matter before it produces a charmonium. For center of mass energy at $\sqrt{s_{N N}}=200 \mathrm{GeV}$, it is known that $f_{p+p}\left(\mathbf{p}_{\mathbf{T}}\right)=\alpha\left(1+p_{T}^{2} / \beta\right)^{-6}$ with $\beta=4.1 \mathrm{GeV}^{2}$ [14], and $\left\langle\delta p_{T}^{2}\right\rangle / \lambda_{g N}=0.1 \mathrm{GeV}^{2} / \mathrm{fm}$ and $\sigma_{\mathrm{abs}}=1.5 \mathrm{mb}$ are used for $\mathrm{Au}+\mathrm{Au}$ collisions [11].

Furthermore, for directly produced $J / \psi$ of high $p_{T}$, they may escape from formed QGP in relativistic heavy ion collisions, leading to the so-called leakage effect.

\section{2 regenerated $J / \psi$}

For regenerated $J / \psi$, their number is similarly determined as in the statistical model, that is

$$
\begin{aligned}
N_{\mathrm{reg} J / \psi}^{A A}= & \gamma^{2}\left\{n_{J / \psi} S_{\mathrm{th}-H}^{J / \psi}+B r\left(\chi_{c} \rightarrow J / \psi\right) n_{\chi_{c}} S_{\mathrm{th}-H}^{\chi_{c}}\right. \\
& \left.+B r\left(\psi^{\prime} \rightarrow J / \psi\right) n_{\psi^{\prime}} S_{\mathrm{th}-H}^{\psi^{\prime}}\right\} V R .
\end{aligned}
$$

In the above, $n_{J / \psi}, n_{\chi_{c}}$, and $n_{\psi^{\prime}}$ are, respectively, the equilibrium densities of $J / \psi, \chi_{c}$, and $\psi^{\prime}$ at chemical freeze-out when the system has volume $V$. The charm fugacity parameter $\gamma$ takes into account the fact that the total charm quark number is not in chemical equilibrium. It is determined from

$$
N_{c \bar{c}}^{A A}=\left\{\frac{1}{2} \gamma n_{0} \frac{I_{1}\left(\gamma n_{0} V\right)}{I_{0}\left(\gamma n_{0} V\right)}+\gamma^{2} n_{h}\right\} V
$$

where $I_{0}$ and $I_{1}$ are modified Bessel functions with $n_{0}$ and $n_{h}$ being, respectively, the densities of open and hidden 
charmed hadrons, and $N_{c \bar{c}}^{A A}$ is the total charm quark pairs produced from initial hard scattering. The latter is given by

$$
N_{c \bar{c}}^{A A}=\sigma_{c \bar{c}}^{N N} A^{2} T_{A A}(\mathbf{b}),
$$

where $\sigma_{c \bar{c}}^{N N}$ is the cross section for $c \bar{c}$ pair production in a $p+p$ collision, which is $63.7 \mu \mathrm{b}$ per unit rapidity near midrapidity at $\sqrt{s}=200 \mathrm{GeV}$ in perturbative QCD [18]. The factor $R$ in Eq. (8) takes into account the nonequilibrium effect of charm quarks in QGP and is evaluated according to

$$
R=1-\exp \left\{-\int_{\tau_{0}}^{\tau_{\mathrm{QGP}}} d \tau \Gamma_{c}(T(\tau))\right\},
$$

where $\tau_{0}$ and $\tau_{\mathrm{QGP}}$ are, respectively, the time charm quark pairs are produced from initial hard scattering and the time QGP phase ends, and $\Gamma_{c}(T)$ is the thermal scattering width of charm quarks in QGP and is calculated using the leadingorder $\mathrm{pQCD}$.

\section{A schematic model for fire-cylinder expansion}

For the dynamics of relativistic heavy-ion collisions, we have used a schematic fire-cylinder model in which the hot dense matter formed in the collision is assumed to be in global thermal equilibrium and evolves boost invariantly in the longitudinal direction but with an accelerated transverse expansion. Specifically, its volume in central heavy ion collisions expands in the proper time $\tau$ according to

$$
V(\tau)=2 \beta\left(\tau_{0}+\tau\right) \pi\left(r_{0}+\frac{1}{2} a_{T} \tau^{2}\right)^{2}
$$

In the above, the factor 2 takes into account the forward and backward expansions in the beam direction, $\beta$ is the longitudinal velocity of nuclear matter, which is approximately equal to the rapidity near midrapidity, $\tau_{0}$ is thermalization time, and $r_{0}$ is the initial radius of QGP on the transverse plane. For the transverse acceleration $a_{T}$ of the firecylinder, it is given by $a_{T}=\left(p-p_{f}\right) A / M$ in terms of the outward force due to the pressure $p$ inside the fire-cylinder on the area $A$ of its cylindrical side divided by the the inertia mass $M$ against its transverse expansion. The $p_{f}$ is the pressure of the fire-cylinder at freeze-out as the transverse acceleration should vanish at freeze-out when particles undergo free streaming afterwards. For the pressure inside the fire-cylinder, it is calculated in the quasiparticle picture [19], which assumes that strongly interacting massless partons (quarks and gluons) can be substituted by noninteracting massive partons to reproduce the energy density and pressure extracted from the lattice QCD, if the matter inside is a QGP. In the case of a hadron gas (HG), the pressure is calculated in the noninteracting resonance gas model of mesons of masses less than $1.5 \mathrm{GeV}$ and baryons of masses less than $2.0 \mathrm{GeV}$. We note that the QGP to HG phase transition is first-order in this model.
The inertia mass $M$ is a free parameter and is determined by fitting the average transverse momenta of light hadrons to experimental data using the light hadron rapidity and transverse momentum distributions,

$$
\frac{d N}{d y d p_{T}^{2}}=\frac{\tau m_{T}}{(2 \pi)^{2}} \int d A_{T} I_{0}\left[\frac{p_{T} \sinh \rho}{T_{\mathrm{f}}}\right] K_{1}\left[\frac{m_{T} \cosh \rho}{T_{\mathrm{f}}}\right],
$$

where $A_{T}$ is the transverse area, $\rho=\tanh \left(v_{T}\right)$ is the transverse rapidity with $v_{T}$ being the transverse velocity in unit of the velocity of light $c$ at the thermal freeze-out temperature $T_{\mathrm{f}}=120 \mathrm{MeV}$, and $I_{n}$ and $K_{n}$ are modified Bessel functions. From measured average transverse momenta of pions, kaons and protons in $\mathrm{Au}+\mathrm{Au}$ collisions at $\sqrt{s}=200$ $\mathrm{GeV}$ and $0-5 \%$ centrality [20], the inertia mass is found to be $M=570 \mathrm{GeV} / c^{2}$.

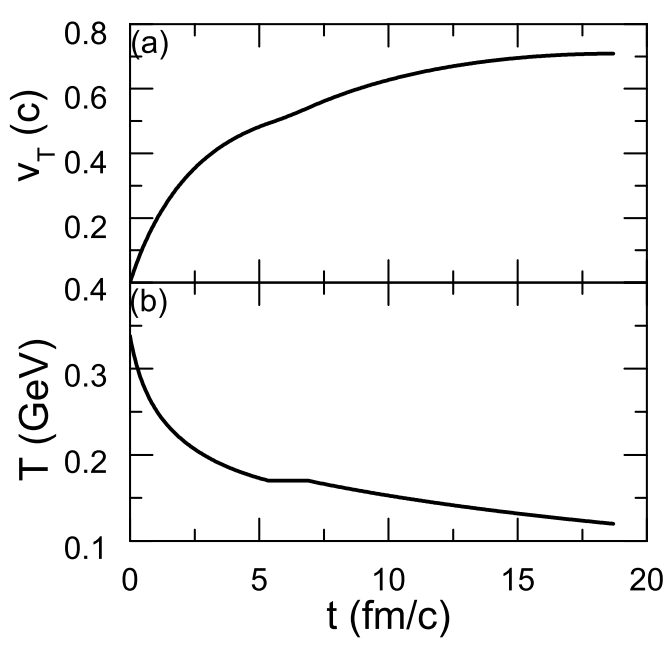

Fig. 1. Transverse velocity (a) and temperature (b) of fire-cylinder in central $\mathrm{Au}+\mathrm{Au}$ collisions at $\sqrt{s}=200 \mathrm{GeV}$ as functions of time.

In Fig. 1(a), we show the resulting transverse velocity of the fire-cylinder obtained with the acceleration as a function of time. The transverse velocity is seen to increase rapidly at first and then slowly. For the temperature of the fire-cylinder, which starts at the initial value of $T_{0}=338$ $\mathrm{MeV}$, is obtained by assuming that the total entropy of the fire-cylinder is conserved during expansion, it decreases with time as shown in Fig. 1(b). It is further seen that the QGP phase terminates at $t=5.4 \mathrm{fm} / c$ when the temperature is $T_{c}=170 \mathrm{MeV}$, the mixed phase at $t=6.9 \mathrm{fm} / c$, and the HG phase reaches the freeze-out temperature at $t=18.7 \mathrm{fm} / c$.

In noncentral collisions, the difference in the length of the overlapping region of two colliding nuclei along the direction of impact parameter and that in its perpendicular direction on the transverse plane, designated, respectively, as the $x$-direction and the $y$-direction, leads to a difference in the pressure gradient in these two directions, resulting in an anisotropic acceleration of the transverse dimension of 
the fire-cylinder. This effect is included by introducing the following accelerations along the $x$ and the $y$ directions:

$$
a_{x}=a_{T}(1+z \epsilon), \quad a_{y}=a_{T}(1-z \epsilon),
$$

in terms of the eccentricity $\epsilon=\left(R_{y}-R_{x}\right) /\left(R_{y}+R_{x}\right)$, where $R_{x}$ and $R_{y}$ are, respectively, the half length of the fire cylinder in the $x$ and $y$ directions on the transverse plane. The constant $z$ in Eq. (14) is determined by requiring that the final $v_{2}$ of light hadrons, determined by the expectation value of $\cos (2 \varphi)$ as

$$
\begin{aligned}
& v_{2}\left(p_{T}\right)= \\
& \frac{\int d A_{T} \cos (2 \phi) I_{2}\left(p_{T} \sinh \rho / T\right) K_{1}\left(m_{T} \cosh \rho / T\right)}{\int d A_{T} I_{0}\left(p_{T} \sinh \rho / T\right) K_{1}\left(m_{T} \cosh \rho / T\right)}
\end{aligned}
$$

reproduces the experimental data.

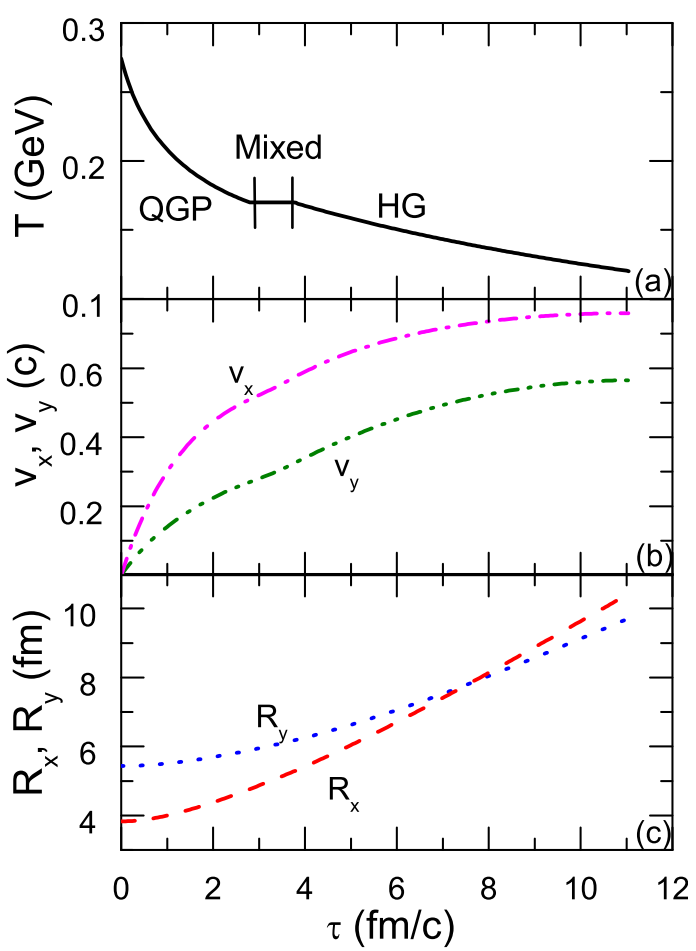

Fig. 2. (color online) Time evolutions of temperature (a), transverse flow velocities $v_{x}$ and $v_{y}$ (b), and transverse radii $R_{x}$ and $R_{y}$ (c) in $\mathrm{Au}+\mathrm{Au}$ collisions at $\sqrt{s}=200 \mathrm{GeV}$ and impact parameter $b=9 \mathrm{fm}$.

In Fig. 2, we show the time evolutions of the temperature $T$, transverse velocities $v_{x}$ and $v_{y}$, and transverse radii $R_{x}$ and $R_{y}$ of the fire-cylinder in $\mathrm{Au}+\mathrm{Au}$ collisions at $\sqrt{s}=200 \mathrm{GeV}$ and impact parameter $b=9 \mathrm{fm}$. These results are obtained with an initial temperature $T_{0}=274$ $\mathrm{MeV}$, the anisotropic constant $z=2.2$ in Eq. (14) and the inertia mass $M=108 \mathrm{GeV} / c^{2}$. It is seen that the QGP phase ends at $t=2.8 \mathrm{fm} / c$, the mixed phase lasts until $t=3.8 \mathrm{fm} / c$, and the freeze-out temperature is reached at $t=11.1 \mathrm{fm} / c$. The flow velocities $v_{x}$ and $v_{y}$ are $0.51 c$ and $0.27 c$ at the phase transition to $\mathrm{HG}$ and reach $0.76 c$ and $0.57 c$ at freeze-out, respectively. We note that the length of $R_{x}$ becomes slightly longer than that of $R_{y}$ at freeze-out.

Using the above parameters for the schematic fire cylinder model, we have reproduced the measured elliptic flow of pions, kaons, and protons from collisions at 20-60\% centrality if we take into account the non-equilibrium effect by multiplying $\exp \left[-C\left(p_{T} / n\right)\right]$ to $\Delta v=\left(v_{x}-v_{y}\right) / 2$, where $C=1.14 c / \mathrm{GeV}$ is a fitting parameter and $n$ is the number of constituent quarks in a hadron. Since the high $p_{T}$ correction factor modifies the Boltzmann distribution, it can be considered as an effective way to include the viscous effect on the fire-cylinder expansion. In the quark-counting picture for hadron scattering, the ratio of the baryon to the meson scattering cross sections is $3 / 2$. This then leads to a smaller viscous effect on baryons than mesons, which is consistent with the quark number dependence in the above high $p_{T}$ correction factor. We have also reproduced the average transverse momenta of pions, kaons, and protons as functions of the number of participants if the inertia mass is parameterized as $M=0.36 N_{\text {part }}^{1.24}$ with $N_{\text {part }}$ being the number of participants in a collision.

\section{Nuclear modification factor of $J / \psi$}

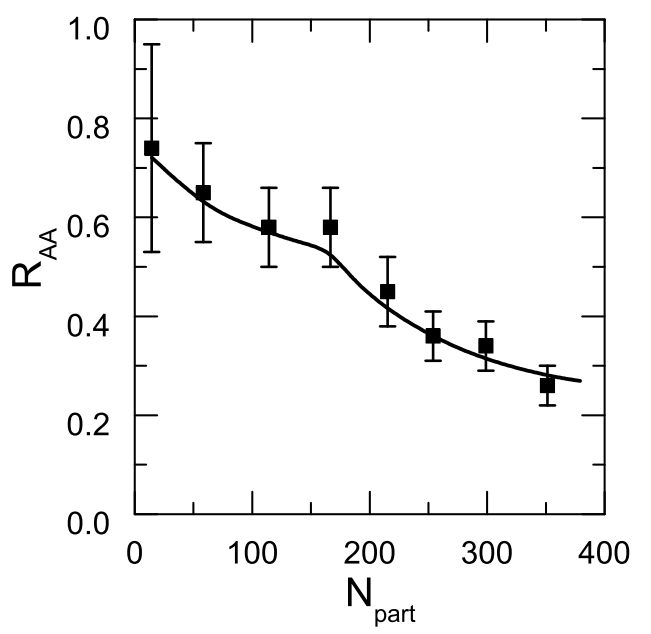

Fig. 3. $R_{A A}$ of $J / \psi$ as a function of the number of participants in $\mathrm{Au}+\mathrm{Au}$ collisions at $\sqrt{s}=200 \mathrm{GeV}$. The experimental data from the PHENIX Collaboration [4] are shown by filled squares.

With the two-component model described in Sec. 2, the expanding fire-cylinder model in Sec. 3, and the thermal freeze-out temperature $T_{\text {cf }}=160 \mathrm{MeV}$ for $J / \psi$, we have studied the $R_{A A}$ of $J / \psi$ as a function of the number of participants. The results are shown in Fig. 3 by the solid line and are seen to reproduce very well the experimental data shown by filled squares [4]. The kink in the theoretical results at $N_{\text {part }} \approx 160$ is caused by the discontinuity 


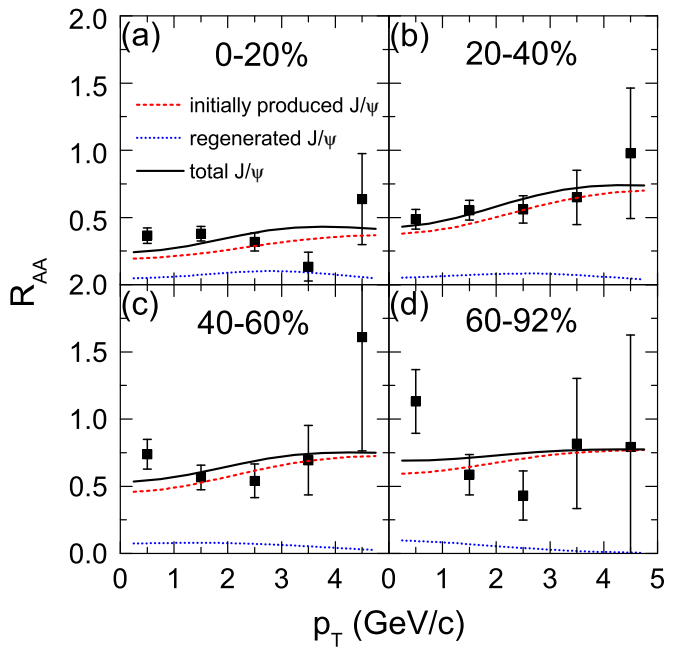

Fig. 4. (color online) Nuclear modification factor $R_{A A}$ of $J / \psi$ as a function of transverse momentum in $\mathrm{Au}+\mathrm{Au}$ collisions of 0 $20 \%$ (a), $20-40 \%$ (b), $40-60 \%$ (c) and $60-92 \%$ (d) centralities at $\sqrt{s}=200 \mathrm{GeV}$. Dashed and dotted lines are, respectively, the $R_{A A}$ from initially produced $J / \psi$ and regenerated $J / \psi$, and the solid line is the sum of the two.

in the survival probability of $J / \psi$ in the region of the firecylinder where the temperature is higher than the dissociation temperature and in the region where the temperature is lower than the dissociation temperature. Since the Cronin effect only changes the transverse momentum of $J / \psi$, not the number of $J / \psi$, it does not affect the $R_{A A}$ of $J / \psi$. The leakage effect, on the other hand, may increase $R_{A A}$ or decrease $R_{A A}$, depending on whether $J / \psi$ initially produced in the corona can move into the fire-cylinder and on whether $J / \psi$ initially produced inside the fire-cylinder can escape to the outside. The net effect due to leakage is, however, small.

Figure 4 shows the $R_{A A}$ of $J / \psi$ as a function of transverse momentum for different centrality bins. It is seen that the $R_{A A}$ of initially produced $J / \psi$, given by the dashed line, increases with transverse momentum because of the Cronin and leakage effects. On the contrary, the $R_{A A}$ of regenerated $J / \psi$ decreases with transverse momentum at high $p_{T}$ as shown by the dotted line.

In Fig. 5, we show the participant number dependence of the average squared transverse momentum of $J / \psi$ in $\mathrm{Au}+\mathrm{Au}$ collisions from the two-component model. Our results reproduce the experimental data for peripheral and mid-central collisions. For central collisions, the two component model overestimates the measured average squared transverse momentum of $J / \psi$. One possible reason for this is that we may have overestimated the diffusion of charm quarks inside the fire-cylinder. The number of charm quarks produced in heavy-ion collision is proportional to the number of binary collisions of nucleons, which is more concentrated in the central region of the fire-cylinder. The number of light quarks is, on the other hand, proportional to the number of participants. Since the relative concentration of the number of binary collisions to the number of participants is larger in central collisions than in semi-central or

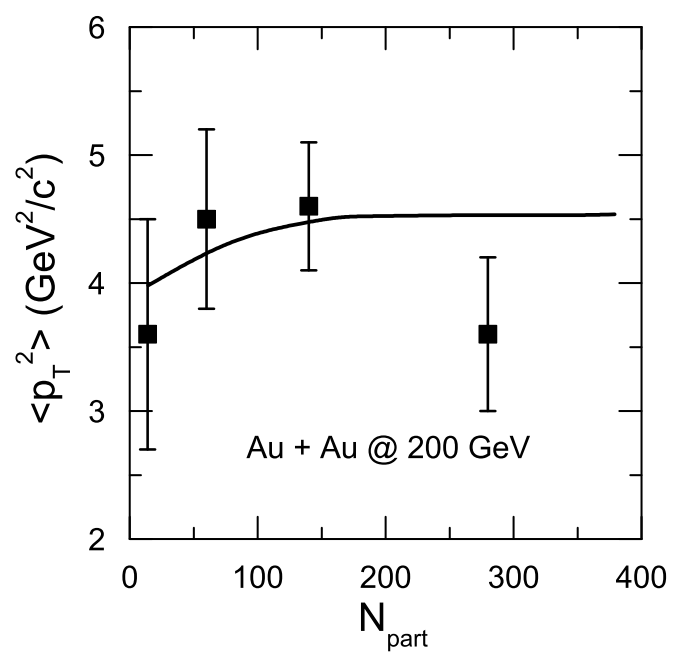

Fig. 5. Average squared transverse momentum of $J / \psi$ in $\mathrm{Au}+\mathrm{Au}$ collisions at $\sqrt{s_{N N}}=200 \mathrm{GeV}$ from the two-component model (solid line) and experimental data (filled squares).

peripheral collisions, charm quarks are initially more concentrated in the central region than light quarks. If the diffusion of charm quarks is slow, they would remain near the central region even at hadronization and their transverse velocity would then be smaller than those of light quarks.

\section{5 elliptic flow of $J / \psi$}

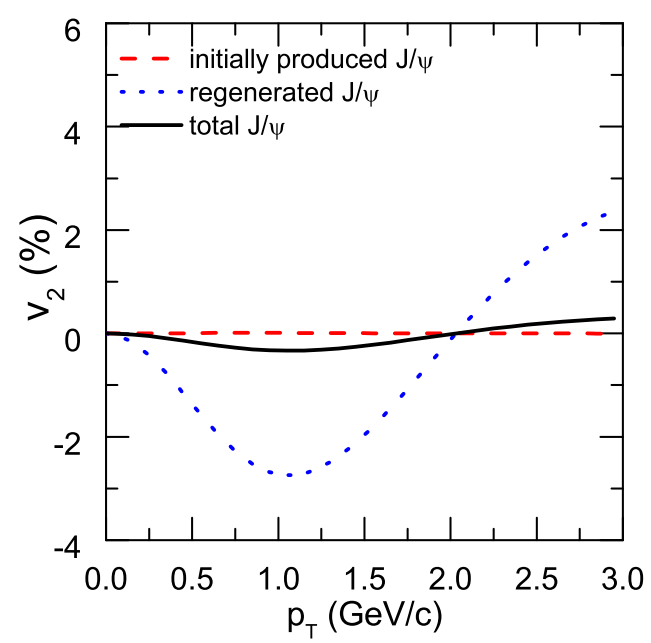

Fig. 6. (color online) Elliptic flow $v_{2}$ of $J / \psi$ for $\mathrm{Au}+\mathrm{Au}$ collisions at $\sqrt{s}=200 \mathrm{GeV}$ and $b=9 \mathrm{fm}$ in the two-component model. The dashed, dotted and solid lines are, respectively, the $v_{2}$ of initially produced, regenerated, and total $J / \psi$.

We have also studied the elliptic flow $v_{2}$ of $J / \psi$ in the two-component model with the high- $p_{T}$ correction to the 
flow velocity as in the case for light hadrons. The results are shown in Fig. 6 for initially produced, regenerated and total $J / \psi$ in $\mathrm{Au}+\mathrm{Au}$ collisions at $\sqrt{s_{N N}}=200 \mathrm{GeV}$ and $b=9 \mathrm{fm}$. It is seen that the $v_{2}$ of initially produced $J / \psi$ is essentially zero, whereas the $v_{2}$ of regenerated $J / \psi$ has a minimum value of -0.028 at $p_{T}=1.1 \mathrm{GeV}$. The $v_{2}$ of total $J / \psi$ is slightly negative, because only $12 \%$ of $J / \psi$ are from regeneration for collisions at $b=9 \mathrm{fm}$ in the twocomponent model. We note that the $v_{2}$ of $J / \psi$ has been recently measured by the PHENIX Collaboration for $\mathrm{Au}+\mathrm{Au}$ collisions at $20-60 \%$ centralities $[21,22]$ and it has a value of $-0.15 \pm 0.12$ between $p_{T}=1$ and $2 \mathrm{GeV}$, which is much more negative than our value.

We note that although initially produced $J / \psi$ have zero $v_{2}$ as their transverse momenta are azimuthally isotropic, they could acquire some finite value after escaping the firecylinder due to the anisotropic expansion of the fire cylinder. In noncentral collisions, the size of the fire-cylinder is shorter in the $x$-direction than in the $y$-direction. As a result, the survival probability of $J / \psi$ moving in the $x$ direction will be higher than that moving in the $y$-direction, leading thus to a positive $v_{2}$ for initially produced $J / \psi$ that have escaped from the fire-cylinder. For the $v_{2}$ of regenerated $J / \psi$, its negative value at low $p_{T}$ and positive value at high $p_{T}$ can be understood as follows. Assuming particles are locally thermalized, they then have a thermal distribution in the local frame. Since the thermal distribution is peaked at $p^{2}=2 T\left(T+\sqrt{T^{2}+m^{2}}\right)$, where $T$ and $m$ are the temperature and mass of the particle, respectively, more massive particles thus peak at higher momenta. If the thermalized matter is boosted with a certain velocity, the peak is shifted to higher momentum in the laboratory frame. With a larger flow velocity in the $x$ than in the $y$ direction in noncentral collisions, the peak of the momentum distribution is thus more shifted in the $x$ direction. As the peak shifts to a higher momentum, the number density at momenta lower than the peak momentum becomes smaller than the number density in the unshifted or less shifted momentum distribution. As a result, the number density of $J / \psi$ with small $p_{T}$ moving in the $x$-direction is smaller than that moving in the $y$-direction, leading thus to a negative $v_{2}$ for $J / \psi$ of low $p_{T}$. This effect is opposite for $J / \psi$ with momenta higher than the peak momentum, so the $v_{2}$ of $J / \psi$ with high $p_{T}$ is positive.

\section{1 effect of higher-order pQCD corrections}

Since the strong coupling constant has been found to be large in lattice QCD up to around twice the critical temperature, the QGP remains nonperturbative below this temperature and higher-order corrections in pQCD may not be negligible. To include higher-order corrections, we have simply introduced some multiplication factors to the dissociation cross sections of charmonia and to the elastic cross section of charm quarks as following:

$$
\begin{aligned}
\sigma_{J / \psi+q(g) \rightarrow c+\bar{c}+X}^{\prime} & =A \sigma_{J / \psi+q(g) \rightarrow c+\bar{c}+X} \\
\sigma_{c+q(g) \rightarrow c+q(g)}^{\prime} & =B \sigma_{c+q(g) \rightarrow c+q(g)},
\end{aligned}
$$

where $A$ and $B$ are arbitrary constants.

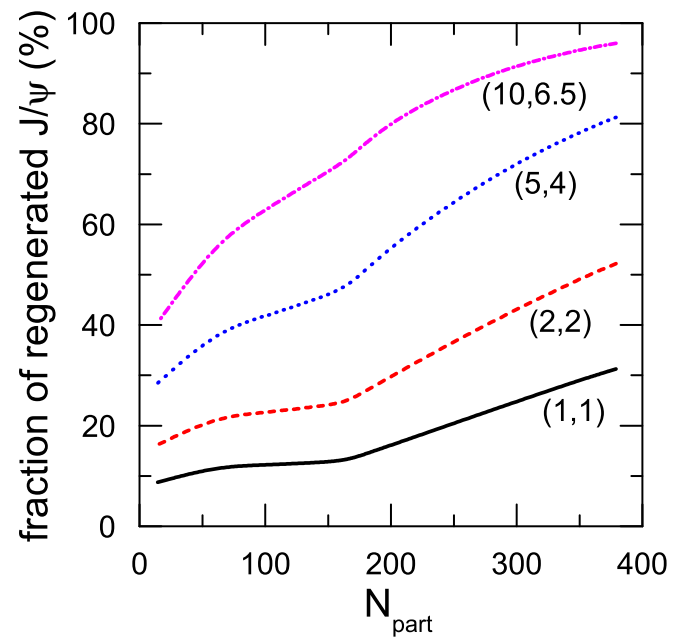

Fig. 7. (color online) Fraction of regenerated $J / \psi$ among total $J / \psi$ in $\mathrm{Au}+\mathrm{Au}$ collisions at $\sqrt{s}=200 \mathrm{GeV}$. Solid, dashed, dotted, and dot-dashed lines correspond, respectively, to multiplicative factors $(\mathrm{A}, \mathrm{B})=(1,1),(2,2),(5,4)$ and $(10,6.5)$ for higher-order corrections.

Figure 7 shows the fraction of regenerated $J / \psi$ among total $J / \psi$ in $\mathrm{Au}+\mathrm{Au}$ collisions at $\sqrt{s}=200 \mathrm{GeV}$ in the two-component model. Without higher-order corrections (solid line), the regenerated $J / \psi$ is about $31 \%$ of total $J / \psi$ produced in central collisions. Including higher-order corrections, the percentage increases to 52,81 and $96 \%$, respectively, for multiplicative factors $(A, B)=(2,2),(5,4)$ and $(10,6.5)$, shown by dashed, dotted, and dot-dashed lines, respectively. We note that the faction of regenerated $J / \psi$ is smaller in semi-central and peripheral collisions than in central collisions.

Higher-order corrections do not change, however, much the value of the $R_{A A}$ of $J / \psi$ even with a large correction factor of ten. This is due to the balance between the dissociation and the regeneration of $J / \psi$, with the suppression of $J / \psi$ caused by enhanced dissociation compensated by the enhancement of regenerated $J / \psi$. Nevertheless, as the correction factor becomes large, the kink in the $R_{A A}$ curve around $N_{\text {part }}=160$ becomes less noticeable as large higher-order corrections reduce the difference in the survival probability of $J / \psi$ in the low and high temperature regions.

The $p_{T}$ dependence of the $R_{A A}$ of $J / \psi$ is also not much affected by higher-order corrections as a result of the large transverse flow of regenerated $J / \psi$. As shown in Fig. 4, the $p_{T}$ dependence of the $R_{A A}$ of initially produced $J / \psi$ is similar to that of regenerated $J / \psi$ except at high $p_{T}$ where the $R_{A A}$ of initially produced $J / \psi$ increases while that of regenerated $J / \psi$ decreases. As a result, the $R_{A A}$ at high $p_{T}$ decreases when higher-order corrections are included, and this seems to contradict with the $p_{T}$ dependence of measured $R_{A A}$, which is an increasing function at high $p_{T}$. Although higher-order corrections are small at high $p_{T}$, they are relatively large at low $p_{T}$, and this seems to reproduce the observed decreasing $R_{A A}$ with increasing $p_{T}$, when $p_{T}$ 
is low, in peripheral collisions. This is mainly due to the short lifetime of the QGP phase in peripheral collisions, so the transverse flow is not sufficiently developed to give a large transverse flow velocity to the regenerated $J / \psi$.

The higher-order corrections are also found to have very little effect on the participant number dependence of the average squared transverse momentum of $J / \psi$.

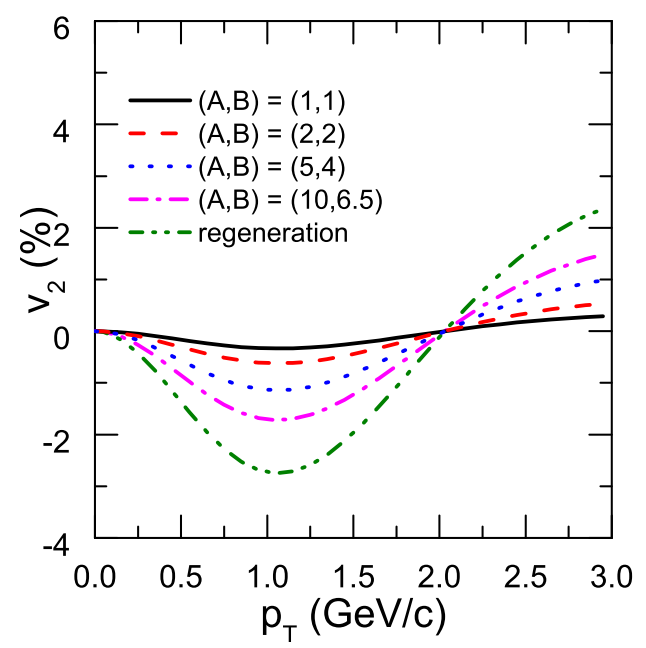

Fig. 8. (color online) Elliptic flow $v_{2}$ of $J / \psi$ in $\mathrm{Au}+\mathrm{Au}$ collisions at $\sqrt{s}=200 \mathrm{GeV}$ and $b=9 \mathrm{fm}$ with higher-order corrections. Solid, dashed, dotted, and dot-dashed lines correspond, respectively, to multiplicative factors $(A, B)=(1,1),(2,2),(5,4)$ and $(10,6.5)$ for the higher-order corrections. The double-dot-dashed line is the $v_{2}$ of $J / \psi$ if they are totally from regeneration.

Different from the $R_{A A}$ of $J / \psi$, the change in $v_{2}$ due to higher-order corrections is significant as shown in Fig. 8 for $\mathrm{Au}+\mathrm{Au}$ collisions at $\sqrt{s}=200 \mathrm{GeV}$ and $b=9 \mathrm{fm}$. It is seen that with increasing multiplicative factors, the $v_{2}$ of $J / \psi$ becomes increasingly more negative at low $p_{T}$ and increasing more positive at high $p_{T}$. We note that for multiplicative factors $(\mathrm{A}, \mathrm{B})=(1,1),(2,2),(5,4)$ and $(10,6.5)$ for the higher-order corrections, the fraction of regenerated $J / \psi$ among total $J / \psi$ is $12 \%, 23 \%, 42 \%$ and $63 \%$, respectively. The double-dot-dashed line in Fig. 8 is the $v_{2}$ of $J / \psi$ if all $J / \psi$ are produced from regeneration. It is worthwhile to point out that even in this extreme case, the $v_{2}$ of $J / \psi$ still only reaches the upper error bar of experimental data, which has a value of -0.03 between $p_{T}=1$ and $2 \mathrm{GeV}$ [21, 22]. If the experimental data on $v_{2}$ of $J / \psi$ is correct, it then means that the fraction of regenerated $J / \psi$ is not small even in semi-central collisions.

\section{6 summary and conclusions}

We have investigated in the two-component model the $R_{A A}$ of $J / \psi$ as a function of transverse momentum and the number of participants as well as the $v_{2}$ of $J / \psi$ in relativistic heavy-ion collisions. The study was based on a schematic model for the expansion of the fire-cylinder formed in these collisions. In this model, the transverse acceleration of the fire-cylinder was assumed to be proportional to the outgoing force given by the product of the pressure of the hot dense matter inside the fire-cylinder and the surface area of the fire-cylinder, and inversely proportional to an inertia mass, which was taken to be a free parameter to fit the experimental data on the transverse momentum spectra of light hadrons. The anisotropic expansion in non-central collisions was modeled by simply adding or subtracting an additional term proportional to the eccentricity of the firecylinder to the acceleration in $x$-direction or $y$-direction. The coefficient of the additional term was determined such that the $v_{2}$ of light hadrons was reproduced after including the high- $p_{T}$ correction to the flow velocity due to the nonequilibrium effect. Both the Cronin effect due to the initial transverse momentum broadening of gluons in nuclei and the leakage effect due to the escape of initially produced $J / \psi$ from produced hot dense matter on the $R_{A A}$ and $v_{2}$ of $J / \psi$ were included. For the kinetic freeze-out temperature of $J / \psi$, it was taken to be the same as the chemical freezeout temperature, because of the much smaller elastic cross section of $J / \psi$ than that of light hadrons, which normally has a lower kinetic freeze-out temperature than the chemical freeze-out temperature. For Au+Au collisions at $\sqrt{s}=$ $200 \mathrm{GeV}$, the model was shown to give a good description of participant number dependence of the $R_{A A}$ of $J / \psi$. It could also describe the increase of $J / \psi R_{A A}$ with increasing transverse momenta as a result of the inclusion of the Cronin and leakage effects in our study. For the $J / \psi$ elliptic flow, its value was found to be small as $J / \psi$ was mostly produced from initial hard scattering, which has essentially zero $v_{2}$.

We have also studied the effect due to higher-order corrections in $\mathrm{pQCD}$ by simply multiplying separate constant factors to the dissociation cross sections of charmonia and to the elastic cross section of charm quarks. The former is related to the thermal decay widths of charmonia and the latter to the relaxation factor of charm quarks. Higherorder corrections were, however, not applied to the screening mass. We found that higher-order corrections enhanced both the dissociation of charmonia and the relaxation of charm quarks. The former decreases the number of initially produced $J / \psi$ while the latter increases the number of regenerated $J / \psi$. As a result, the total number of $J / \psi$ was found to change very little after including higherorder corrections. However, the fraction of initially produced $J / \psi$ and that of regenerated ones were affected significantly by higher-order corrections with larger higherorder corrections leading to a larger fraction of regenerated $J / \psi$ among total $J / \psi$. This is the reason why the $R_{A A}$ of $J / \psi$ as a function of the number of participants was insensitive to the higher-order corrections, and both the two-component model and the statistical model can describe successfully the dependence of $R_{A A}$ on the number of participants. Applying same higher-order corrections to the study of the transverse momentum dependence of the $R_{A A}$ of $J / \psi$, we found that it was also insensitive to the fraction of regenerated $J / \psi$ among the total $J / \psi$ except at high $p_{T}$, as the small transverse momentum of regen- 
erated $J / \psi$ is compensated by the large transverse flow velocity. On the other hand, the $v_{2}$ of $J / \psi$ is sensitive to the fraction of regenerated $J / \psi$ with increasing more negative value at low $p_{T}$ and more positive value at high $P_{T}$. In our study, we have assumed that regenerated $J / \psi$ are produced from thermalized charm quarks in QGP. It is not clear how close charm quarks are from thermal equilibrium in heavy-ion collisions. Recent experimental data on nonphotonic single electrons, which are produced through the decay of heavy mesons, suggest that charm quarks are significantly thermalized [23]. If charm quarks are thermalized, resulting thermally produced $J / \psi$ would share their thermal properties and thus acquire appreciable $v_{2}$. Also, if there is an abrupt decrease in the mass of charmonia near $T_{c}$ as suggested in Ref. [24], the yield of regenerated $J / \psi$ would be enhanced and so is its contribution to $v_{2}$.

We have used in the present study an equation of state with a first-order phase transition between the strongly coupled quark-gluon plasma and the hadronic resonance gas. Recent lattice QCD calculations imply, however, that the QCD phase transition is a crossover in the high temperature and low net baryon density matter produced in heavyion collisions at RHIC. Although a crossover transition would increase the duration of the transition between the quark-gluon plasma and the hadronic matter, it is not expected to affect much our results as the quark-gluon plasma has a smaller average volume during the crossover phase transition than during the first-order phase transition, resulting thus in a small difference in the four volume.

Presently, the $v_{2}$ of $J / \psi$ has large error bars in the experimental data. If refined and precise data on the $v_{2}$ of $J / \psi$ become available, they are expected to play an important role in discriminating between the two production mechanisms for $J / \psi$, i.e., initial production and regeneration.
7. L. Grandchamp and R. Rapp, Nucl. Phys. A709, (2002) 415

8. L. Yan, P. Zhuang and N. Xu, Phys. Rev. Lett. 97, (2006) 232301

9. A. Andronic, P. Braun-Munzinger, K. Redlich and J. Stachel, Nucl. Phys. A789, (2007) 334

10. T. Song, W. Park and S. H. Lee, Phys. Rev. C 81, (2010) 034914

11. X. Zhao and R. Rapp, Phys. Lett. B664, (2008) 253

12. S. V. Akkelin, P. Braun-Munzinger and Yu. M. Sinyukov, Phys. Rev. C 81, (2010) 034912

13. T. Song, C. M. Ko, S. H. Lee, and J. Xu, arXiv:1008.2730 [nucl-th]; Phys. Rev. C, in press.

14. A. Adare et al. [PHENIX Collaboration], Phys. Rev. Lett. 98, (2007) 232002

15. Y. Park, K. I. Kim, T. Song, S. H. Lee and C. Y. Wong, Phys. Rev. C 76, (2007) 044907

16. T. Song, Y. Park, S. H. Lee and C. Y. Wong, Phys. Lett. B659, (2008) 621

17. L. A. Linden Levy [PHENIX Collaboration], J. Phys. G 36, (2009) 064013

18. M. Cacciari, P. Nason and R. Vogt, Phys. Rev. Lett. 95, (2005) 122001

19. P. Levai and U. W. Heinz, Phys. Rev. C 57, (1998) 1879

20. S. S. Adler et al. [PHENIX Collaboration], Phys. Rev. C 69, (2004) 034909

21. C. Silvestre [PHENIX Collaboration], J. Phys. G 35, (2008) 104136

22. D. Krieg and M. Bleicher, Eur. Phys. J. A 39, (2009) 1

23. A. Adare et al. [PHENIX Collaboration], Phys. Rev. Lett. 98, (2007) 172301

24. K. Morita and S. H. Lee, Phys. Rev. Lett. 100, (2008) 022301

\section{Acknowledgements}

This talk was based on work supported in part by the U.S. National Science Foundation under the Grant No. PHY0758115, the Welch Foundation under Grant No. A-1358, the Korean Ministry of Education through the BK21 Program, and the Korea Research Foundation under Grant No. KRF-2006-C00011.

\section{References}

1. T. Matsui and H. Satz, Phys. Lett. B178, (1986) 416

2. M. C. Abreu et al. [NA50 Collaboration], Phys. Lett. B410, (1997) 337

3. R. Arnaldi et al. [NA60 Collaboration], Nucl. Phys. A 783, (2007) 261

4. A. Adare et al. [PHENIX Collaboration], Phys. Rev. Lett. 98, (2007) 232301

5. S. S. Adler et al. [PHENIX Collaboration], Phys. Rev. Lett. 96, (2006) 012304

6. B. Zhang, C. M. Ko, B. A. Li, Z. w. Lin and B. H. Sa, Phys. Rev. C 62, (2000) 054905 